\title{
Differences in the Gut Microbiome of Women With and Without Hypoactive Sexual Desire Disorder: Case Control Study
}

Guanjian $\mathrm{Li}^{1^{*}}$, MD; Weiran $\mathrm{Li}^{2^{*}}, \mathrm{MD}, \mathrm{PhD}$; Bing Song ${ }^{3^{*}}, \mathrm{MD}, \mathrm{PhD}$; Chao Wang ${ }^{4 *}, \mathrm{MD}$; Qunshan Shen ${ }^{5^{*}}$, MD; Bo $\mathrm{Li}^{6}, \mathrm{MD}, \mathrm{PhD}$; Dongdong Tang ${ }^{7}, \mathrm{MD}$; Chuan $\mathrm{Xu}^{2}, \mathrm{MD}$; Hao Geng ${ }^{7}$; Yang $\mathrm{Gao}^{3}$, MD; Guanxiong Wang ${ }^{3}$, MD; Huan $\mathrm{Wu}^{3}$, MD; Zhiguo Zhang ${ }^{1}$, MD, PhD; Xiaofeng Xu ${ }^{7}$, MD, PhD; Ping Zhou ${ }^{3}, \mathrm{MD}, \mathrm{PhD}$; Zhaolian Wei ${ }^{5}, \mathrm{MD}, \mathrm{PhD}$; Xiaojin $\mathrm{He}^{4^{*}}, \mathrm{MD}, \mathrm{PhD}$; Yunxia $\mathrm{Cao}^{1 *}, \mathrm{MD}, \mathrm{PhD}$

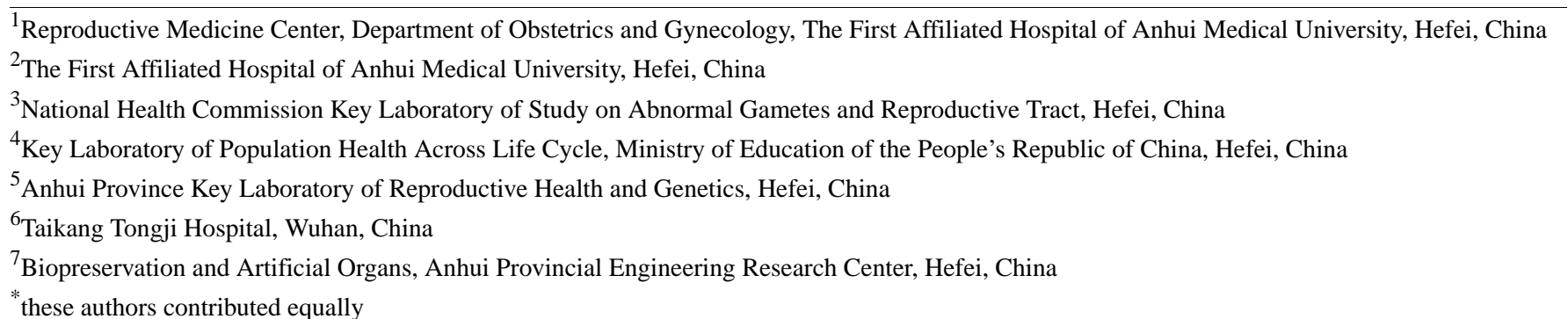

Corresponding Author:

Yunxia Cao, $\mathrm{MD}, \mathrm{PhD}$

Reproductive Medicine Center

Department of Obstetrics and Gynecology

The First Affiliated Hospital of Anhui Medical University

81 Meishan Road

Hefei,

China

Phone: 8615395104659

Email: caoyunxia6@126.com

\section{Abstract}

Background: The gut microbiome is receiving considerable attention as a potentially modifiable risk factor and therapeutic target for numerous mental and neurological diseases.

Objective: This study aimed to explore and assess the difference in the composition of gut microbes and fecal metabolites between women with hypoactive sexual desire disorder (HSDD) and healthy controls.

Methods: We employed an online recruitment method to enroll "hard-to-reach" HSDD populations. After a stringent diagnostic and exclusion process based on DSM-IV criteria, fecal samples collected from 24 women with HSDD and 22 age-matched, healthy controls underwent microbiome analysis using $16 \mathrm{~S}$ ribosomal RNA gene sequencing and metabolome analysis using untargeted liquid chromatography-mass spectrometry.

Results: We found a decreased abundance of Ruminococcaceae and increased abundance of Bifidobacterium and Lactobacillus among women with HSDD. Fecal samples from women with HSDD showed significantly altered metabolic signatures compared with healthy controls. The abundance of Bifidobacterium, Lactobacillus, and several fecal metabolites correlated negatively with the sexual desire score, while the number of Ruminococcaceae correlated positively with the sexual desire score in all subjects.

Conclusions: Our analysis of fecal samples from women with HSDD and healthy controls identified significantly different gut microbes and metabolic signatures. These preliminary findings could be useful for developing strategies to adjust the level of human sexual desire by modifying gut microbiota.

Trial Registration: Chinese Clinical Trial Registry ChiCTR1800020321; http://www.chictr.org.cn/showproj.aspx?proj=34267

(J Med Internet Res 2021;23(2):e25342) doi: 10.2196/25342 


\section{KEYWORDS}

gut microbiome; metabolome; sexual desire; online recruitment; biomarkers

\section{Introduction}

Hypoactive sexual desire disorder (HSDD) is defined as a deficiency or absence of desire for sexual activity that causes marked distress or interpersonal difficulty and is not accounted for by another psychiatric condition, use of medications, or relationship problems [1,2]. Two large epidemiological surveys have shown that this combination of symptoms, "low desire and associated distress," is present in up to $10 \%$ of American women, with similar prevalence rates seen throughout the world $[3,4]$. HSDD is the most frequently reported female sexual health problem, and women with HSDD may experience reduced quality of life, impaired physical image, and decreased feelings of self-confidence and self-worth, in addition to feeling less connected with their partners and families [5].

In humans, sexual desire is regulated by key areas of the brain through the action of various neurotransmitters [6,7]. Norepinephrine, dopamine, melanocortin, oxytocin, and vasopressin mediate sexual excitation, while serotonin, opioids, prolactin, and the endogenous cannabinoid system mediate sexual inhibition. Generalized HSDD may be related to a neuropsychological state of increased inhibition or decreased excitation, or a mixture of the two [8,9]. Neurotransmitters in the central nervous system are thus therapeutic targets for improving human sexual desire. At present, the main treatment strategies are to reduce the action of 5-HT, enhance the action of dopamine, or both [10].

Human beings exist in a mutualistic relationship with their gut microbiota, a highly diverse and complex microbial ecosystem closely associated with various phenotypes and diseases $[11,12]$. Perturbations in gut microbe richness and diversity affect the levels of 5-HT, norepinephrine, and $\gamma$-aminobutyric acid (GABA)ergic and dopaminergic neurotransmission in the brain [13-15]. These pathways and molecules are believed to be closely related to human sexual desire.

Although this evidence suggests that there may be a connection between the composition of gut microbiota and sexual desire, our knowledge of the role of gut microbiota in sexual desire disorders is limited. We hypothesized that the fecal gut microbiota and metabolites of patients with sexual desire disorders differ from those of people without such disorders and that active metabolites and neurochemicals may be mediators between gut microbes and the sexual desire system.

Recruiting participants for microbial research is increasingly difficult as the number of projects competing for participants' attention increases and response rates decline. In addition, women with desire disorder constitute a hard-to-reach population owing to fear and lack of trust in the study procedures or in research staff, especially in a more conservative society like China when compared with Western countries [16]. Widespread access to online tools now offers various advantages in data collection that can reduce administrative procedures and improve information privacy $[17,18]$. Previous studies have demonstrated that performance and effectiveness obtained using online recruitment methods in studies of sexual issues or gut microbes concur with those obtained using offline recruitment methods, such as telephone surveys, mail-in questionnaires, posters, and flyers [12,19-21]. In addition, online surveys may even provide greater reach and be less expensive, while allowing for convenient data collection and analysis [22-24]. These findings suggest that the growth of the internet and social media sites may provide new opportunities for research on the correlation between sensitive health concerns (such as mental health problems or sexual health disorders) and gut microbes.

We conducted this study to investigate the composition of the gut microbiome and metabolite abundance in women with HSDD and to increase our understanding of the possible association between human sexual desire and gut microbiota and fecal metabolites.

\section{Methods}

\section{Participants}

From March 2019 to November 2019, we recruited subjects by posting advertisements on instant messenger applications and the information platform. In all advertisements, potential participants were automatically redirected to a study website that included a link to the survey and participant information including study details and information statement.

Potential recruits included women who had previously been diagnosed with suspected HSDD but not yet treated or who complained of decreased sexual desire, causing them to seek medical counseling, as well as volunteers who claimed to have normal sexual desire. No incentives were offered to responders. When requested, they were provided a brief report on their own gut microbial composition. All responders provided electronic written informed consent. All experimental protocols used in this study were approved by the Ethics Committee of Anhui Medical University and the relevant hospitals.

\section{Clinical Information Collection}

We collected information online from 157 premenopausal women and scheduled face-to-face diagnostic interviews and biological sample collection to identify women with generalized acquired HSDD. Incomplete questionnaires and responses that rejected further face-to-face interviews were not accepted by the system. A combination of a web-based questionnaire and structured interviews was used to collect information on demographic characteristics such as ethnicity, marital status, income, educational level, reproductive history, sexual partner relationships, sexual frequency, sexual dysfunction, and the presence and treatment of related diseases.

\section{S Ribosomal RNA Gene Sequencing and Metabolomics Tests}

Subsequently, we performed high-throughput 16S ribosomal RNA gene sequencing and untargeted liquid chromatography-mass spectrometry metabolomic analysis of stool samples from the 26-member HSDD cohort and 26 healthy 
controls (for all survey measures and analysis methods, see the Methods section in Multimedia Appendix 1).

\section{Results}

\section{Clinical Characteristics of the Subjects}

A flowchart showing the inclusion and exclusion processes is presented in Figure 1. In the end, 24 women with HSDD and 22 women with no history of sexual dysfunction (NHSD) were included in the final analyses. The clinicopathological variables of the HSDD and NHSD cohorts are shown in Table 1. All participants were Han Chinese from the Hefei area and had comparable geographical conditions and eating habits. The clinicopathological variables of the HSDD and NHSD cohorts were comparable, except for a slight increase in platelet count in the HSDD cohort (the meaning is unclear). Significant differences were observed in Female Sexual Function Index desire domain scores between the HSDD and NHSD groups.

Figure 1. Inclusion and exclusion processes. HSDD: hypoactive sexual desire disorder; LC-MS: liquid chromatography-mass spectrometry; NHSD: no history of sexual dysfunction.

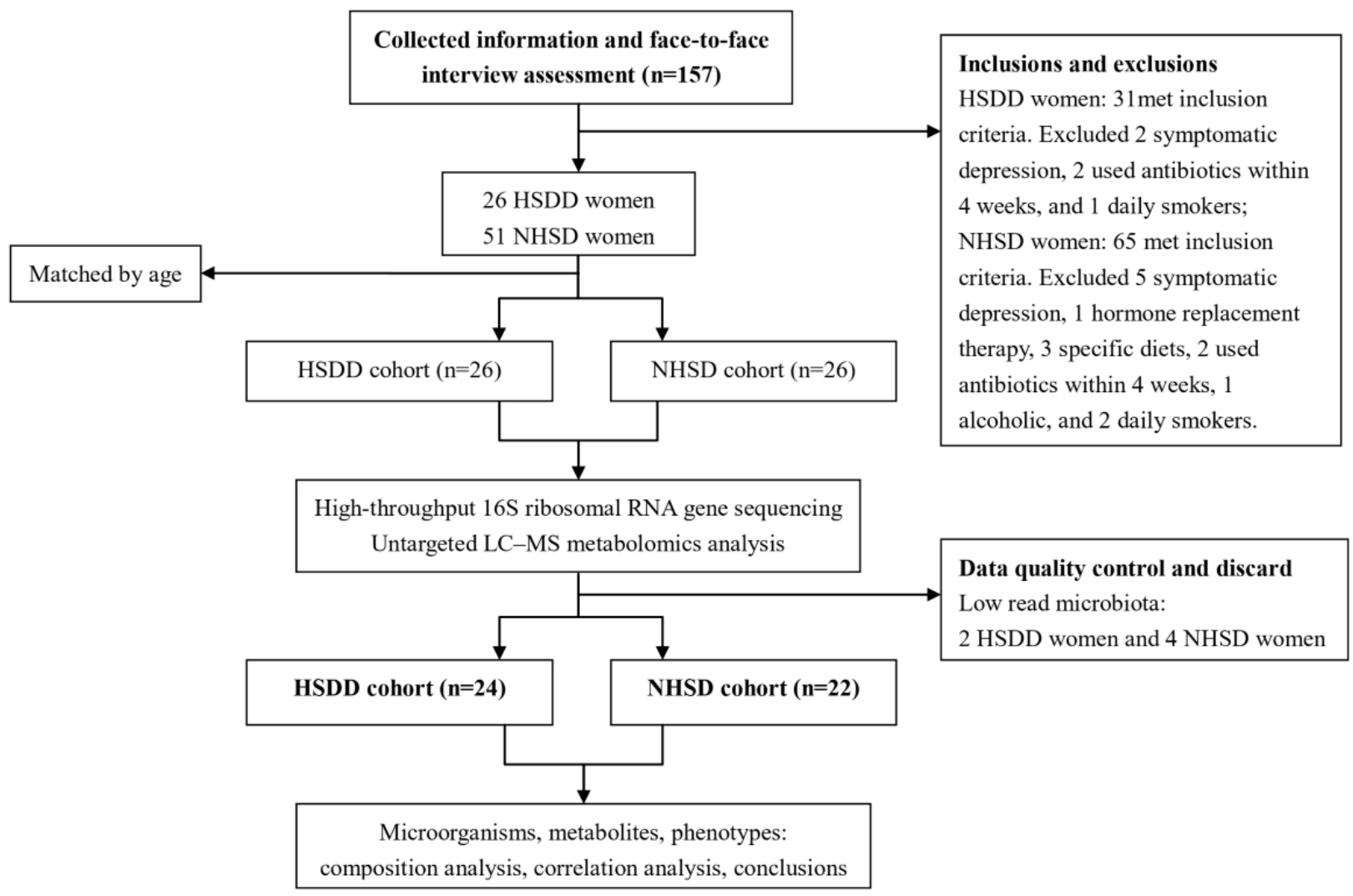


Table 1. Demographic and other sample data at baseline.

\begin{tabular}{|c|c|c|c|}
\hline Characteristic & $\operatorname{HSDD}^{\mathrm{a}}(\mathrm{n}=24)$ & $\operatorname{NHSD}^{\mathrm{b}}(\mathrm{n}=22)$ & $P$ value \\
\hline Age (years), mean (SD) & $33.8(4.5)$ & $33.9(4.0)$ & .91 \\
\hline BMI $\left(\mathrm{kg} / \mathrm{m}^{2}\right)$, mean $(\mathrm{SD})$ & $27.1(3.6)$ & $26.3(3.3)$ & .27 \\
\hline College or beyond, $\mathrm{n}$ & 17 & 16 & .89 \\
\hline FSFI-D ${ }^{c}$ score, mean (SD) & $3.2(0.9)$ & $8.6(1.0)$ & .01 \\
\hline CES-D ${ }^{\mathrm{d}}$ score, mean (SD) & $10.3(4.9)$ & $10.9(4.6)$ & .55 \\
\hline $\mathrm{RBC}^{\mathrm{e}}\left(\times 10^{12} / \mathrm{L}\right)$, mean $(\mathrm{SD})$ & $4.3(0.7)$ & $4.1(0.4)$ & .06 \\
\hline $\mathrm{WBC}^{\mathrm{f}}\left(\times 10^{9} / \mathrm{L}\right)$, mean $(\mathrm{SD})$ & $6.9(1.7)$ & $6.5(1.7)$ & .28 \\
\hline $\mathrm{PLT}^{\mathrm{g}}\left(\times 10^{12} / \mathrm{L}\right)$, mean $(\mathrm{SD})$ & $169.4(44.8)$ & $154.0(35.9)$ & .04 \\
\hline $\mathrm{FSH}^{\mathrm{h}, \mathrm{i}}(\mathrm{mIU} / \mathrm{mL})$, mean $(\mathrm{SD})$ & $6.3(3.0)$ & $6.3(2.9)$ & .93 \\
\hline $\mathrm{LH}^{\mathrm{i}, \mathrm{j}}(\mathrm{mIU} / \mathrm{mL})$, mean $(\mathrm{SD})$ & $5.3(3.4)$ & $5.9(3.7)$ & .67 \\
\hline Estradiol $^{\mathrm{i}}(\mathrm{pg} / \mathrm{mL})$, mean $(\mathrm{SD})$ & $48.1(9.3)$ & $50.6(6.5)$ & .09 \\
\hline Total $\mathrm{T}^{\mathrm{i}, \mathrm{k}}(\mathrm{ng} / \mathrm{mL})$, mean (SD) & $0.5(0.3)$ & $0.5(0.2)$ & .86 \\
\hline
\end{tabular}

${ }^{a}$ HSDD: hypoactive sexual desire disorder.

bHSD: no history of sexual dysfunction.

${ }^{c}$ FSFI-D: Female Sexual Function Index desire domain.

${ }^{\mathrm{d}}$ CES-D: Center for Epidemiologic Studies Depression Scale.

${ }^{\mathrm{e}} \mathrm{RBC}$ : red blood cell.

${ }^{f}$ WBC: white blood cell.

g $_{\text {PLT: platelet. }}$

${ }^{\mathrm{h}} \mathrm{FSH}$ : follicle-stimulating hormone.

${ }^{\mathrm{i}}$ Due to missing data, the group sizes differ for hormonal data: HSDD group, $\mathrm{n}=20$; NHSD group, $\mathrm{n}=14$.

${ }^{\mathrm{j}} \mathrm{LH}$ : luteinizing hormone.

$\mathrm{k}_{\mathrm{T}}$ : testosterone.

\section{HSDD and Gut Microorganisms}

In our investigation, 1753 operational taxonomic units were identified from sequenced specimens with $97 \%$ sequence similarity. Different operational taxonomic unit-based diversity indexes were used to assess the bacterial composition within each sample (the $\alpha$-diversity). Compared to the NHSD group, the HSDD cohort was characterized by a higher number of observed species and higher Shannon and Chao 1 indexes
(Figure 2A, except for Simpson), suggesting greater species diversity in gut microbiota in the HSDD cohort. $\beta$-diversity analysis was performed to determine whether HSDD was associated with altered gut microbial composition. As shown in Figure 2B, both unweighted and weighted principal coordinate analysis plots revealed that the gut microbial composition of the HSDD group was significantly different from that of the NHSD group. 
Figure 2. Gut microbial characteristics of women with hypoactive sexual desire disorder (HSDD) or with no history of sexual dysfunction (NHSD), based on (A) $\alpha$-diversity analysis of 3 indexes (observed species, shannon, and Chao 1); (B) $\beta$-diversity analysis, providing unweighted and weighted principal coordinate (PC) plots; (C) component proportion of bacterial phylum in each group; (D) the ratio between HSDD and NHSD on the logarithmic scale. All QFDR $<0.05$.

A

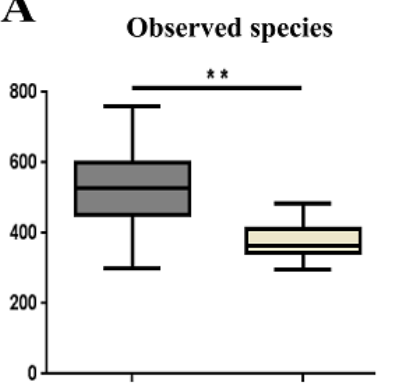

HSDD $(n=24) \quad$ NHSD $(n=22)$

B

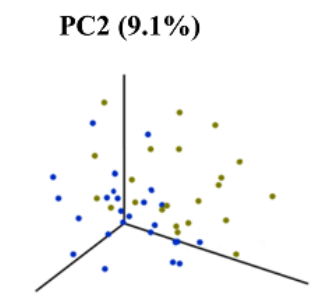

PC3 (5.8\%) PC1 (14.6\%)

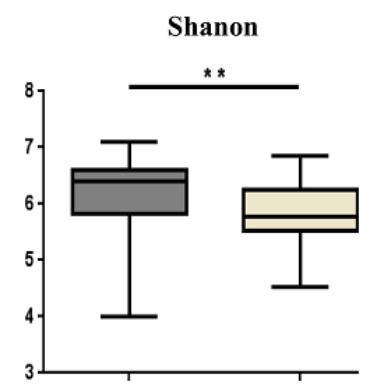

HSDD $(n=24) \quad$ NHSD $(n=22)$

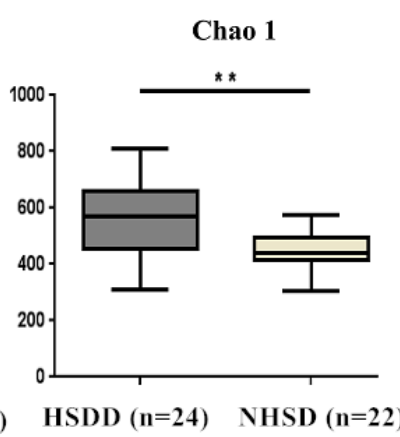

C 1.

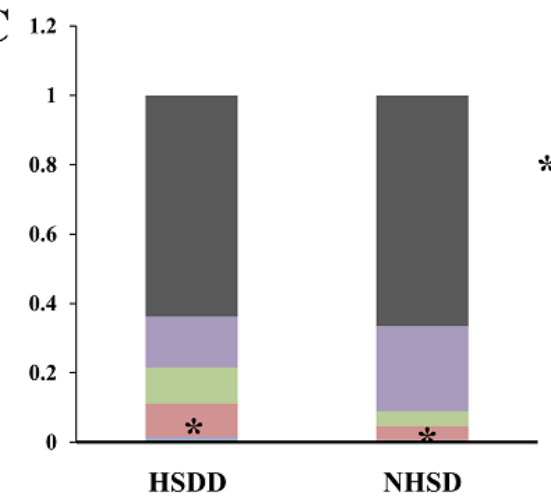

Simpson

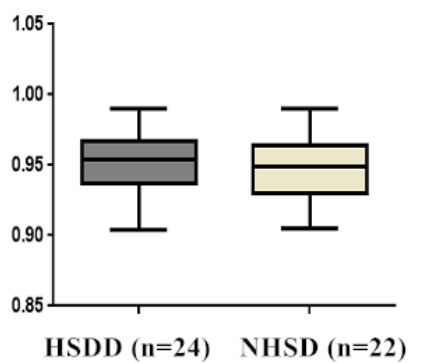

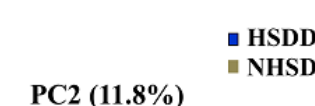

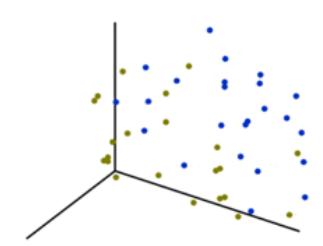

PC2 $(7.3 \%)$

PC2 (41.4\%)

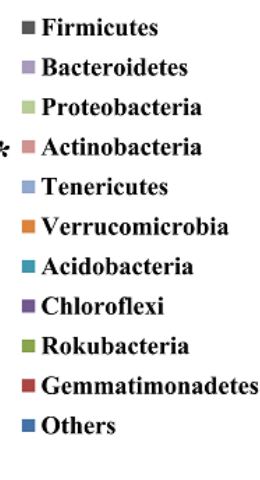

airmicutes

Bacteroidetes

Proteobacteria

Actinobacteria

Tenericute

Verrucomicrobia

Acidobacteria

Chloroflexi

Rokubacteria

Gemmatimonadetes

NHSD

D

\begin{tabular}{llll} 
Order & Family & Genus \\
\hline Sphingomonadales & Sphingomonadaceae & Porphyrobacter \\
Corynebacteriales & Corynebacteriaceae & unidentified_Corynebacteriaceae & Massilia \\
unidentified_Gammaproteobacteria & Burkholderiaceae & unidentified_Actinobacteria \\
unidentified_Actinobacteria & unidentified_Actinobacteria & Sphingomonas \\
Sphingomonadales & Sphingomonadaceae & Phenylobacterium \\
Caulobacterales & Caulobacteraceae & unidentified_Ruminococcaceae & \\
Clostridiales & Ruminococcaceae & Bifidobacterium & \\
Bifidobacteriales & Bifidobacteriaceae & Lactobacillus & \\
Lactobacillales & Lactobacillaceae & 0.01 & 0.1 \\
\hline
\end{tabular}

At the phylum level, the most abundant taxa were Firmicutes and Bacteroidetes, followed by Proteobacteria and Actinobacteria in the exploration cohort (Figure 2C). Actinobacteria were significantly enriched in the HSDD group compared to the NHSD group $(9.1 \%$ versus $3.3 \%, P<.01)$. At the genus level, 9 taxa were different between the HSDD and NHSD groups (Figure 2D). Bifidobacterium, Lactobacillus, and Sphingomonas were enriched in women with HSDD, while an unidentified genus of Ruminococcaceae was enriched in NHSD women $(P<.05$ and QFDR $<0.20)$. Lactobacillus and Ruminococcaceae belong to the same phylum, Firmicutes, which is the most abundant phylum, while Bifidobacterium belong to the phylum Actinobacteria.

\section{HSDD and the Fecal Metabolome}

In the fecal metabolome analysis, 1168 metabolites were quantified. Differential metabolite volcanic maps show that the fecal metabolic phenotype of women with HSDD was significantly different than that of women with NHSD (Figure 3A). Principal component analysis clearly showed differences between the HSDD and NHSD groups, indicating that women with HSDD have specific metabolic abundances and characteristics (Figure 3B). In total, 28 metabolites that made major contributions to the distinction (VIP score >1.5) were selected using partial least squares discriminant analysis. These differential metabolites were annotated to 12 different KEGG metabolic pathways [25], including neuroactive ligand-receptor interactions, the Fc epsilon RI signaling pathway, steroid 
hormone biosynthesis, histidine metabolism, and tryptophan metabolism (Figure 3C).

Figure 3. Fecal metabolites characteristics of women with hypoactive sexual desire disorder (HSDD) and women with no history of sexual dysfunction (NHSD), displayed as (A) volcano plots showing the differentially accumulated (log2[fold change] and significantly changed (-log10[pvalue] fecal metabolites; (B) principal component (PC) analysis showing grouped discrimination; (C) functions and pathways of these metabolites using the KEGG database, where the differential metabolites are annotated on 12 different KEGG metabolic pathways; (D) Lefse and linear discriminant analyses showing differences in taxonomic composition; (E) receiver operating characteristic (ROC) curve. AUC: area under the curve; TRP: tryptophan.

A
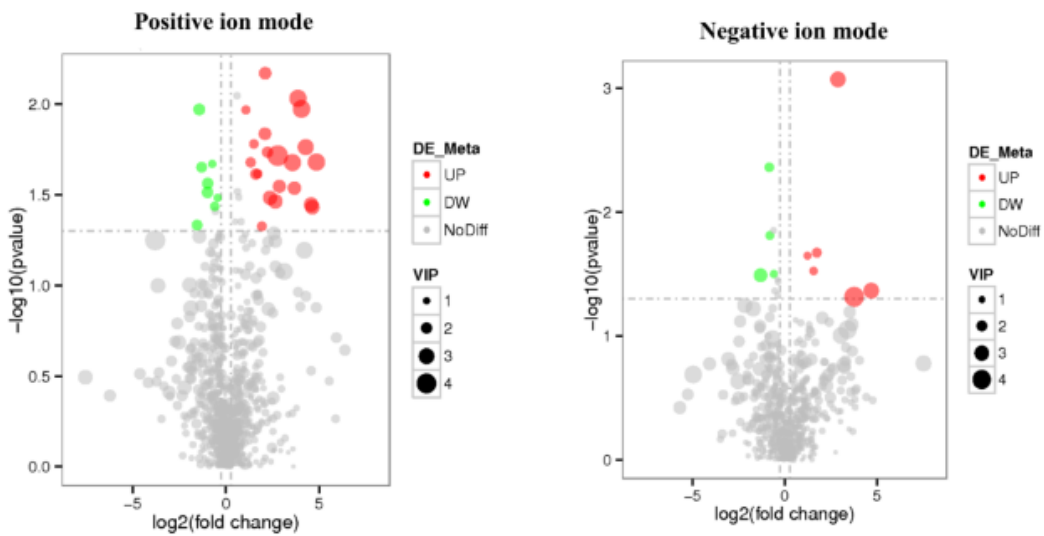

B

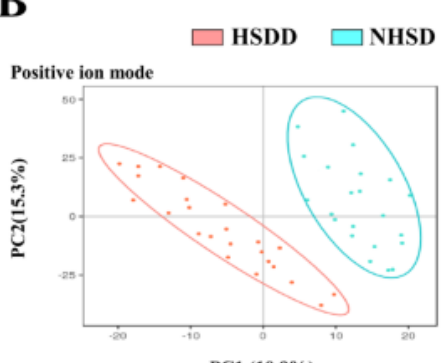

C

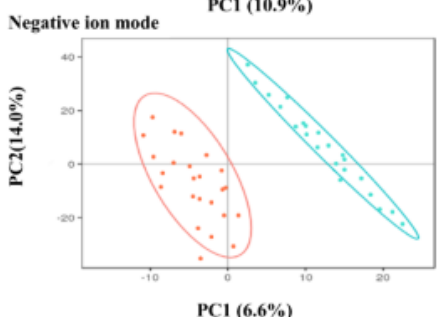

Fc epsilon RI signaling pathway

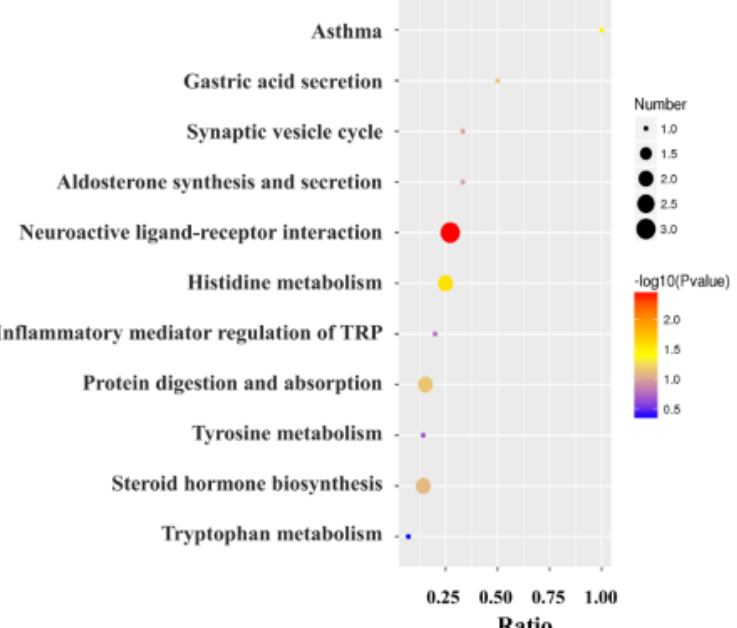

$\mathbf{E}$

D

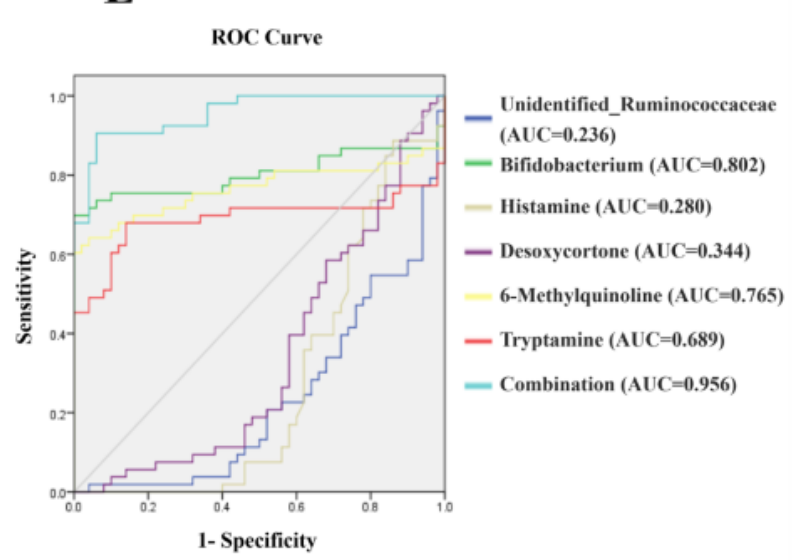




\section{A Panel of Fecal Microorganisms and Metabolic Markers Identifies HSDD}

To further identify the specific bacterial components associated with HSDD, we used a standard microbiota analysis tool, LefSe; 8 taxa were identified as key biomarkers $(P<.05$, linear discriminant analysis scores $>4$ ). Several taxa, including the genus Bifidobacterium and class unidentified Actinobacteria, were significantly overrepresented in the HSDD women, whereas the genus unidentified Ruminococcaceae was overrepresented in the NHSD women (Figure 3D).

To identify and quantify the potential of microbial and metabolic biomarkers for HSDD, we performed a receiver operating characteristic curve analysis using the relative abundance of the Bifidobacterium genus and the unidentified_Ruminococcaceae genus, the most abundant genus identified, to distinguish HSDD from NHSD. Then, a binary logistic regression analysis for the 2 genera and 28 significantly correlated metabolites was performed. Significant deviations between women with HSDD and women with NHSD could be identified using Bifidobacterium and unidentified_Ruminococcaceae at the genus level, as well as 4 significantly correlated metabolites: histamine $(P=.01)$, desoxycortone $(P=.03)$, 6-Methylquinoline $(P=.01)$, and tryptamine $(P=.03)$. This diagnostic marker panel (combination of 2 microbial markers and 4 fecal metabolites) discriminated between the HSDD and NHSD groups with $95.6 \%$ accuracy (area under the curve $=0.956$; Figure 3E).

\section{Relationships Between Gut Microbiota, Metabolites, and Clinical Characteristics}

A Spearman's rank correlation test was performed to explore the correlations between gut microbes, fecal metabolites, and clinical characteristics. The altered bacterial genera were generally associated with differential metabolites (Multimedia Appendix 2), and almost all the differential bacterial genera showed significant correlations with a range of differential fecal metabolites $(P<.05)$. Spearman correlation analysis between every 2 variables suggested that some bacterial genera and fecal metabolomes were slightly associated with clinical characteristics such as age, BMI, Center for Epidemiologic Studies Depression Scale score, and some blood indicators (Multimedia Appendix 2). We found that some genera and metabolites were significantly correlated with the Female Sexual Function Index desire domain score $(|\mathrm{r}|>0.35, P<.05)$, providing strong evidence for the existence of a correlation between sexual desire and gut microorganisms.

\section{Discussion}

\section{Principal Findings}

These results suggest significant differences in the composition of fecal microbiota between controls and women with HSDD. We observed a significantly higher quantity of Actinobacteria in women with HSDD compared with healthy controls. The altered abundance of several genera contributed to the unique gut microbial characteristics found in HSDD. The most prominent of these was the marked enrichment of the Bifidobacterium genus. Correlation analysis showed that the greater number of Bifidobacterium and Lactobacillus could be associated with a decrease in sexual desire. Higher microbial diversity has been shown in previous studies to have benefits for the human body, and elevated levels of Bifidobacterium and Lactobacillus have been related to reduced anger, dysphoric mood, aggressive thoughts, and self-reported feelings of sadness $[26,27]$. A possible explanation for these observations is that these emotions and human sexual desires are physiologically interrelated, involving a variety of neurotransmitters and other changes in brain biochemistry. Anger, fear, or stress can be a prelude to sex, specifically because these emotional states generate enthusiasm or excitement that leads to an "excitement transfer" into sexual desire and arousal [28].

There is considerable evidence that gut microorganisms modulate the metabolism of dopamine, 5-HT, and noradrenaline in the brain $[15,29,30]$. These molecules are generally considered to be the major neurotransmitters regulating sexual desire [7]. Previous research suggests that plasma concentrations of kynurenic acid and tryptophan (serotonergic precursor) in Sprague-Dawley rats treated with Bifidobacterium for 14 days increased significantly compared with controls. Bifidobacterium treatment also resulted in a decrease in 5-hydroxyindoleacetic acid concentration in the frontal cortex and dihydroxyphenylacetic acid concentration in the amygdala [31]. In another study, rats were orally administered specific Bifidobacterium every day, and Bifidobacterium reportedly produced GABA through glutamate decarboxylase [32]. Ruminococcaceae, also commonly referred to as Clostridia clusters XIVa and IV, are abundant microorganisms in the normal gut. They decompose indigestible carbohydrates and produce short-chain fatty acids (SCFAs). Numerous studies have shown that SCFAs, including butyrate, can cross the blood-brain barrier and enter the central nervous system and may play a role in neuropsychiatric conditions and general psychological functions [33]. SCFAs might directly influence the brain by influencing the blood-brain barrier, stimulating the vagus nerve, regulating the secretion of neurotrophic factors, modulating 5-HT/dopamine/noradrenaline/GABA biosynthesis, and promoting the transcription of inhibitory pathway transcripts [34]. These findings lead to the hypothesis that neurotransmitter molecules and pathways may play crucial roles in the regulation of sexual desire by gut microorganisms.

The analysis of fecal metabolic components has received considerable attention since these biomolecules reflect genetic and environmental effects and also act to connect the health of the host and its symbiotic microorganisms [35]. In fecal samples from women with HSDD, metabolites had significantly different distributions from the control group. Enriched functional modules included those involved in neuroactive ligand-receptor interactions, histidine metabolism, steroid hormone biosynthesis, and tryptophan metabolism.

The systematic exclusion of alternative diagnoses by structured interviews and the use of rigorous differential diagnosis criteria based on the DSM-IV resulted in a representative cohort of individuals with generalized acquired HSDD, avoiding various genital disorders existing in men or elderly women, rather than the sexual desire problem of "central effect." In addition, although we reported Center for Epidemiologic Studies Depression Scale 20 scores, estradiol, testosterone, and sex 
hormone-binding globulin, in fact, the cause of HSDD is multifactorial and variable; changes in hormones, stress, depression, present sexual experiences, past history of abuse, total well-being, sexual condition of the sexual partner, and relationship factors may also be crucial causes of sexual desire disorder [36,37]. Therefore, whether our findings might be specific to HSDD or more general changes seen in multiple disorders need to be further explored in future studies with large samples.

Given the increased popularity of online media and information platforms, many researchers are using web-based tools to recruit participants for medical, psychological, and sociological research studies (especially for stigmatized and hard-to-reach groups). Previous studies have shown that online methods can successfully recruit and manage people who are humiliated or discriminated against because of race, sexual orientation, or mental health status [17,18]. Our results confirmed recent literature regarding online recruitment of hard-to-reach people and expanded the scope of recruitment and management to the study population of sexual dysfunction and gut microbe research.

\section{Limitations}

There are several potential limitations of our research. First, our online recruitment methods relied on convenience samples, and determining the source population limits the external validity of findings. When it comes to sensitive issues, determining adequate compensation and ensuring confidentiality continue to be important concerns. Second, gut microorganisms and metabolites are affected by a variety of factors, including geography, daily diet, and genetic variation [38]. Additionally, race and ethnic culture appear to have a significant association with the occurrence of sexual problems [39]. Therefore, since all the participants were from a single ethnicity, identifying specific correlations between gut microorganisms and sexual desire in a global population may not be straightforward. Another issue is that there is no standardized method for adjusting the composition of the microbiome using diet. In this study, this issue was handled by excluding all individuals who reported variations in their diet, such as binge eating or purging, following a vegetarian diet, or having gluten or lactose intolerance. However, it cannot be ruled out that subtle, unspecified dietary factors could have influenced our results. Finally, our findings only demonstrated an association between the gut microbiome and HSDD. Whether alternations in the gut microbiome are responsible for HSDD should be further validated using animal models in the laboratory.

\section{Conclusions}

This study addressed a number of gaps. We identified numerous microbial genera, including Bifidobacterium and Lactobacillus, that were enriched in women with HSDD. In addition, our metabolomic analysis of fecal samples from women with HSDD and healthy controls identified significantly different metabolic signatures. Our results support the hypothesis that disturbances in gut microorganisms are closely related to the level of human sexual desire. Pathogenicity of the microbiome in women with HSDD may be caused by comprehensive changes in holistic interaction, rather than by specific pathogens or metabolites.

A better understanding of the microbiota's role in the gut-brain-libido axis may lead to the identification of novel drug targets and management measures to improve sexual health. Much work remains to be performed in determining the role and mechanisms of the gut microbiota in human sexual desire.

\section{Acknowledgments}

The authors would like to thank all participants. We also thank the researchers from Anhui Medical University, as well as engineers, nurses, and doctors. This work was supported by the Non-profit Central Research Institute Fund of Chinese Academy of Medical Sciences (grant number 2019PT310002).

\section{Conflicts of Interest}

None declared.

\section{Multimedia Appendix 1}

Supplementary methods. [DOCX File, 28 KB-Multimedia Appendix 1]

\section{Multimedia Appendix 2}

Heat map. (a) Heat map of the Spearman's rank correlation coefficient of differential bacterial genus, differential metabolites and HSDD clinical characteristics. (b) Heatmaps showing altered bacterial genus were generally associated with differential fecal metabolites. The statistical significance was denoted on the squares $(\# p<0.05$; \#\#<0.01).

[PNG File, 2362 KB-Multimedia Appendix 2]

\section{References}

1. Diagnostic and statistical manual of mental disorders: DSM-IV. Washington DC: American Psychiatric Association; 1994.

2. Meyers M, Margraf J, Velten J. Psychological Treatment of Low Sexual Desire in Women: Protocol for a Randomized, Waitlist-Controlled Trial of Internet-Based Cognitive Behavioral and Mindfulness-Based Treatments. JMIR Res Protoc 2020 Sep 29;9(9):e20326 [FREE Full text] [doi: 10.2196/20326] [Medline: 32990248] 
3. Shifren JL, Monz BU, Russo PA, Segreti A, Johannes CB. Sexual problems and distress in United States women: prevalence and correlates. Obstet Gynecol 2008 Nov;112(5):970-978. [doi: 10.1097/AOG.0b013e3181898cdb] [Medline: 18978095]

4. West SL, D'Aloisio AA, Agans RP, Kalsbeek WD, Borisov NN, Thorp JM. Prevalence of low sexual desire and hypoactive sexual desire disorder in a nationally representative sample of US women. Arch Intern Med 2008 Jul 14;168(13):1441-1449. [doi: 10.1001/archinte.168.13.1441] [Medline: 18625925$]$

5. Kingsberg SA. Attitudinal survey of women living with low sexual desire. J Womens Health (Larchmt) 2014 Oct;23(10):817-823. [doi: 10.1089/jwh.2014.4743] [Medline: 25105910]

6. Georgiadis JR, Kringelbach ML, Pfaus JG. Sex for fun: a synthesis of human and animal neurobiology. Nat Rev Urol 2012 Sep;9(9):486-498. [doi: 10.1038/nrurol.2012.151] [Medline: 22926422]

7. Pfaus JG. Pathways of sexual desire. J Sex Med 2009 Jun;6(6):1506-1533. [doi: 10.1111/j.1743-6109.2009.01309.x] [Medline: 19453889$]$

8. Toates F. An integrative theoretical framework for understanding sexual motivation, arousal, and behavior. J Sex Res 2009;46(2-3):168-193. [doi: 10.1080/00224490902747768] [Medline: 19308842]

9. Goldstein I, Kim NN, Clayton AH, DeRogatis LR, Giraldi A, Parish SJ, et al. Hypoactive Sexual Desire Disorder: International Society for the Study of Women's Sexual Health (ISSWSH) Expert Consensus Panel Review. Mayo Clin Proc 2017 Jan;92(1):114-128 [FREE Full text] [doi: 10.1016/j.mayocp.2016.09.018] [Medline: 27916394]

10. Stahl SM. Targeting circuits of sexual desire as a treatment strategy for hypoactive sexual desire disorder. J Clin Psychiatry $2010 \mathrm{Jul}$;1(7):821-822 [FREE Full text] [doi: 10.4088/JCP.10bs06117blu] [Medline: 20667289]

11. Uittenbogaart M, Leclercq WK, Bonouvrie D, Romeijn MM, Luijten AA, Olde Damink SW, et al. Diet-Induced Alteration of Microbiota and Development of Obesity, Nonalcoholic Fatty Liver Disease, and Diabetes: Study Protocol of a Prospective Study. JMIR Res Protoc 2019 Jun 19;8(6):e11553 [FREE Full text] [doi: 10.2196/11553] [Medline: $\underline{31219051]}$

12. Kindleysides S, Kruger R, Douwes J, Tannock GW, Renall N, Slater J, et al. Predictors Linking Obesity and the Gut Microbiome (the PROMISE Study): Protocol and Recruitment Strategy for a Cross-Sectional Study on Pathways That Affect the Gut Microbiome and Its Impact on Obesity. JMIR Res Protoc 2019 Aug 26;8(8):e14529 [FREE Full text] [doi: 10.2196/14529] [Medline: 31452525]

13. Mayer EA, Tillisch K, Gupta A. Gut/brain axis and the microbiota. J Clin Invest 2015 Mar 02;125(3):926-938 [FREE Full text] [doi: 10.1172/JCI76304] [Medline: 25689247]

14. González-Arancibia C, Urrutia-Piñones J, Illanes-González J, Martinez-Pinto J, Sotomayor-Zárate R, Julio-Pieper M, et al. Do your gut microbes affect your brain dopamine? Psychopharmacology (Berl) 2019 May;236(5):1611-1622. [doi: 10.1007/s00213-019-05265-5] [Medline: 31098656]

15. Clarke G, Grenham S, Scully P, Fitzgerald P, Moloney RD, Shanahan F, et al. The microbiome-gut-brain axis during early life regulates the hippocampal serotonergic system in a sex-dependent manner. Mol Psychiatry 2013 Jun;18(6):666-673 [FREE Full text] [doi: 10.1038/mp.2012.77] [Medline: 22688187]

16. Ho PSY, Jackson S, Cao S, Kwok C. Sex With Chinese Characteristics: Sexuality Research in/on 21st-Century China. J Sex Res 2018;55(4-5):486-521. [doi: 10.1080/00224499.2018.1437593] [Medline: 29521527]

17. Russomanno J, Patterson JG, Jabson Tree JM. Social Media Recruitment of Marginalized, Hard-to-Reach Populations: Development of Recruitment and Monitoring Guidelines. JMIR Public Health Surveill 2019 Dec 02;5(4):e14886 [FREE Full text] [doi: 10.2196/14886] [Medline: $\underline{\text { 31789598] }}$

18. Ibarra JL, Agas JM, Lee M, Pan JL, Buttenheim AM. Comparison of Online Survey Recruitment Platforms for Hard-to-Reach Pregnant Smoking Populations: Feasibility Study. JMIR Res Protoc 2018 Apr 16;7(4):e101 [FREE Full text] [doi: 10.2196/resprot.8071] [Medline: 29661751]

19. Burns J, Johnstone K, Chavanduka T, Jamison C, Pena V, Stephenson R, et al. Evaluation of the Sexual Health Behaviors of Black Male Adolescents and Young Adults Through Social Media Platforms: Web-Based Survey Study. JMIR Public Health Surveill 2020 Sep 22;6(3):e19219 [FREE Full text] [doi: 10.2196/19219] [Medline: 32693387]

20. Motoki Y, Miyagi E, Taguri M, Asai-Sato M, Enomoto T, Wark JD, et al. Comparison of Different Recruitment Methods for Sexual and Reproductive Health Research: Social Media-Based Versus Conventional Methods. J Med Internet Res 2017 Mar 10;19(3):e73 [FREE Full text] [doi: 10.2196/jmir.7048] [Medline: 28283466]

21. Duncan DT, Park SH, Hambrick HR, Dangerfield Ii DT, Goedel WC, Brewer R, et al. Characterizing Geosocial-Networking App Use Among Young Black Men Who Have Sex With Men: A Multi-City Cross-Sectional Survey in the Southern United States. JMIR Mhealth Uhealth 2018 Jun 14;6(6):e10316 [FREE Full text] [doi: 10.2196/10316] [Medline: 29903702]

22. Christensen T, Riis AH, Hatch EE, Wise LA, Nielsen MG, Rothman KJ, et al. Costs and Efficiency of Online and Offline Recruitment Methods: A Web-Based Cohort Study. J Med Internet Res 2017 Mar 01;19(3):e58 [FREE Full text] [doi: 10.2196/jmir.6716] [Medline: 28249833]

23. Honka AM, Helander E, Pavel M, Jimison H, Mustonen P, Korhonen I, et al. Exploring Associations Between the Self-Reported Values, Well-Being, and Health Behaviors of Finnish Citizens: Cross-Sectional Analysis of More Than 100,000 Web-Survey Responses. JMIR Ment Health 2019 Apr 22;6(4):e12170 [FREE Full text] [doi: 10.2196/12170] [Medline: $\underline{31008710}$ ] 
24. Savolainen I, Oksanen A, Kaakinen M, Sirola A, Paek H. The Role of Perceived Loneliness in Youth Addictive Behaviors: Cross-National Survey Study. JMIR Ment Health 2020 Jan 02;7(1):e14035 [FREE Full text] [doi: 10.2196/14035] [Medline: 31895044]

25. KEGG PATHWAY Database. 2021. URL: https://www.genome.jp/kegg/pathway.html [accessed 2021-02-03]

26. Messaoudi M, Lalonde R, Violle N, Javelot H, Desor D, Nejdi A, et al. Assessment of psychotropic-like properties of a probiotic formulation (Lactobacillus helveticus R0052 and Bifidobacterium longum R0175) in rats and human subjects. Br J Nutr 2011 Mar;105(5):755-764. [doi: 10.1017/S0007114510004319] [Medline: 20974015]

27. Steenbergen L, Sellaro R, van Hemert S, Bosch JA, Colzato LS. A randomized controlled trial to test the effect of multispecies probiotics on cognitive reactivity to sad mood. Brain Behav Immun 2015 Aug;48:258-264 [FREE Full text] [doi: 10.1016/j.bbi.2015.04.003] [Medline: 25862297]

28. Bancroft J, Janssen E, Strong D, Carnes L, Vukadinovic Z, Long JS. The relation between mood and sexuality in heterosexual men. Arch Sex Behav 2003 Jun;32(3):217-230. [doi: 10.1023/a:1023409516739] [Medline: 12807294]

29. Diaz Heijtz R, Wang S, Anuar F, Qian Y, Björkholm B, Samuelsson A, et al. Normal gut microbiota modulates brain development and behavior. Proc Natl Acad Sci U S A 2011 Feb 15;108(7):3047-3052 [FREE Full text] [doi: 10.1073/pnas.1010529108] [Medline: 21282636]

30. Cryan JF, Dinan TG. Mind-altering microorganisms: the impact of the gut microbiota on brain and behaviour. Nat Rev Neurosci 2012 Oct;13(10):701-712. [doi: 10.1038/nrn3346] [Medline: 22968153]

31. Desbonnet L, Garrett L, Clarke G, Bienenstock J, Dinan TG. The probiotic Bifidobacteria infantis: An assessment of potential antidepressant properties in the rat. J Psychiatr Res 2008 Dec;43(2):164-174. [doi: 10.1016/j.jpsychires.2008.03.009] [Medline: 18456279 ]

32. Pokusaeva K, Johnson C, Luk B, Uribe G, Fu Y, Oezguen N, et al. GABA-producing Bifidobacterium dentium modulates visceral sensitivity in the intestine. Neurogastroenterol Motil 2017 Jan;29(1):1 [FREE Full text] [doi: 10.1111/nmo.12904] [Medline: 27458085]

33. Puertollano E, Kolida S, Yaqoob P. Biological significance of short-chain fatty acid metabolism by the intestinal microbiome. Curr Opin Clin Nutr Metab Care 2014 Mar;17(2):139-144. [doi: 10.1097/MCO.0000000000000025] [Medline: 24389673]

34. Kratsman N, Getselter D, Elliott E. Sodium butyrate attenuates social behavior deficits and modifies the transcription of inhibitory/excitatory genes in the frontal cortex of an autism model. Neuropharmacology 2016 Mar;102:136-145. [doi: 10.1016/j.neuropharm.2015.11.003] [Medline: 26577018]

35. Weir TL, Manter DK, Sheflin AM, Barnett BA, Heuberger AL, Ryan EP. Stool microbiome and metabolome differences between colorectal cancer patients and healthy adults. PLoS One 2013;8(8):e70803 [FREE Full text] [doi: 10.1371/journal.pone.0070803] [Medline: 23940645]

36. Varfi N, Rothen S, Jasiowka K, Lepers T, Bianchi-Demicheli F, Khazaal Y. Sexual Desire, Mood, Attachment Style, Impulsivity, and Self-Esteem as Predictive Factors for Addictive Cybersex. JMIR Ment Health 2019 Jan 21;6(1):e9978 [FREE Full text] [doi: 10.2196/mental.9978] [Medline: 30664470]

37. Edmonds MR, Hadjistavropoulos HD, Gullickson KM, Asmundson AJ, Dear BF, Titov N. Understanding Problems With Sleep, Sexual Functioning, Energy, and Appetite Among Patients Who Access Transdiagnostic Internet-Delivered Cognitive Behavioral Therapy for Anxiety and Depression: Qualitative Exploratory Study. JMIR Form Res 2020 Oct 13;4(10):e15037 [FREE Full text] [doi: 10.2196/15037] [Medline: 33048054]

38. He Y, Wu W, Zheng H, Li P, McDonald D, Sheng H, et al. Regional variation limits applications of healthy gut microbiome reference ranges and disease models. Nat Med 2018 Oct;24(10):1532-1535. [doi: 10.1038/s41591-018-0164-x] [Medline: $\underline{30150716]}$

39. Graziottin A. Prevalence and evaluation of sexual health problems--HSDD in Europe. J Sex Med 2007 Mar;4 Suppl 3:211-219. [doi: 10.1111/j.1743-6109.2007.00447.x] [Medline: 17394593]

\author{
Abbreviations \\ GABA: $\gamma$-aminobutyric acid \\ HSDD: hypoactive sexual desire disorder \\ NHSD: no history of sexual dysfunction \\ SCFA: short-chain fatty acids
}


Edited by R Kukafka; submitted 28.10.20; peer-reviewed by B Ma, S Yang; comments to author 10.11.20; accepted 20.01.21; published 25.02.21

Please cite as:

Li G, Li W, Song B, Wang C, Shen Q, Li B, Tang D, Xu C, Geng H, Gao Y, Wang G, Wu H, Zhang Z, Xu X, Zhou P, Wei Z, He X, Cao $Y$

Differences in the Gut Microbiome of Women With and Without Hypoactive Sexual Desire Disorder: Case Control Study

J Med Internet Res 2021;23(2):e25342

URL: https://www.jmir.org/2021/2/e25342

doi: $\underline{10.2196 / 25342}$

PMID: 33629964

(C) Guanjian Li, Weiran Li, Bing Song, Chao Wang, Qunshan Shen, Bo Li, Dongdong Tang, Chuan Xu, Hao Geng, Yang Gao, Guanxiong Wang, Huan Wu, Zhiguo Zhang, Xiaofeng Xu, Ping Zhou, Zhaolian Wei, Xiaojin He, Yunxia Cao. Originally published in the Journal of Medical Internet Research (http://www.jmir.org), 25.02.2021. This is an open-access article distributed under the terms of the Creative Commons Attribution License (https://creativecommons.org/licenses/by/4.0/), which permits unrestricted use, distribution, and reproduction in any medium, provided the original work, first published in the Journal of Medical Internet Research, is properly cited. The complete bibliographic information, a link to the original publication on http://www.jmir.org/, as well as this copyright and license information must be included. 